\title{
NOTAS SOBRE EL INFORME DEL INSTITUTO DE DERECHO EUROPEO ACERCA DE LA PROTECCION DE ADULTOS EN SITUACIONES INTERNACIONES
}

\section{NOTES ON THE REPORT OF THE EUROPEAN LAW INSTITUTE THE PROTECTION OF ADULTS IN INTERNATIONAL SITUATIONS}

\author{
Alberto Muñoz FernándeZ \\ Profesor Contratado Doctor \\ Universidad de Navarra
}

Recibido: 26.06.2020 / Aceptado: 08.07.2020

DOI: https://doi.org/10.20318/cdt.2020.5657

\begin{abstract}
Resumen: El Instituto de Derecho Europeo (ELI) ha elaborado un Informe con el que urge a la Unión Europea a "europeizar" un nuevo ámbito del Derecho internacional privado: la protección de adultos. El Informe razona la necesidad de dar este paso, justifica la base competencial de la UE para hacerlo y propone una doble estrategia, externa e interna. La externa consiste en autorizar a los Estados Miembros a ratificar el Convenio de La Haya sobre protección internacional de adultos en interés de la UE y en promoverlo entre terceros Estados. La interna pasa por la adopción de un Reglamento que complemente y mejore, en las relaciones entre Estados Miembros, el funcionamiento del mencionado Convenio.

Palabras clave: Instituto de Derecho Europeo, protección internacional de adultos, Convenio de La Haya sobre protección internacional de adultos, mandatos de protección.
\end{abstract}

Abstract: The European Law Institute (ELI) adopted a Report encouraging the European Union to "Europeanize" a new field of private international law: the protection of adults. The Report provides arguments on the need to take this step, it justifies the EU's competence to do so and it proposes a double strategy, external and internal. The external one is to authorize the Member States to ratify The Hague Convention on international protection of adults in the interest of the EU and to promote it among third States. The internal action would be the adoption of a Regulation that complements and improves, in the relations between Member States, the functioning of the Convention.

Keywords: European Law Institute, international protection of adults, Hague Convention on International Protection of Adults, mandates of protection.

Sumario: I. Introducción. II. La UE tiene el deber de involucrarse en la protección internacional de adultos. III. Bases competenciales para actuar. IV. Estrategia externa. V. Estrategia interna. VI. Contenido de la Propuesta de Reglamento. VII. Otras propuestas de interés. VIII. Sugerencias para la discusión con vistas a la Comisión especial sobre el Convenio de Adultos. IX. Conclusiones.

\section{Introducción}

1. El Instituto de Derecho Europeo (ELI en sus siglas inglesas) es una organización independiente que tiene como misión mejorar la calidad del derecho europeo. Para ello, promueve la investigación a través de distintos proyectos con el objetivo poder hacer recomendaciones y ofrecer orientación 
práctica en el ámbito del desarrollo del derecho europeo en un sentido amplio, no limitado al derecho de la Unión Europea y el Consejo de Europa.

2. El último de los proyectos concluido por el Instituto lleva por título "Protección de adultos en situaciones vulnerables"1. Su resultado final ha sido un Informe, aprobado el 21 de marzo de 2020, que reviste gran interés y cuyos aspectos principales se comentarán en estas líneas.

3. El Informe aborda, principalmente, las siguientes cuestiones: a) la justificación de la necesidad de acometer, con urgencia, acciones en este sector por parte de la UE; b) el análisis de las distintas bases competenciales de la UE para afrontar la cuestión; c) las distintas estrategias, de acción exterior y de acción interior, que podría emprender la UE; d) una exposición detallada de una propuesta de Reglamento europeo que podría complementar al Convenio de La Haya de 2000 sobre protección internacional de adultos (en adelante, $\mathrm{CH} / 2000$ ); e) una lista de cuestiones a tener en cuenta a la hora de otorgar un mandato de protección. A continuación, se comentarán alguno de estos puntos.

\section{La UE tiene el deber de involucrarse en la protección internacional de adultos}

4. El equipo encargado de llevar a cabo el Informe era consciente de que, pese a los distintos llamamientos hechos por la UE, 20 años después de su adopción, solo un tercio de los Estados miembros son parte actualmente en el $\mathrm{CH} / 2000^{2}$. Y no solo eso, sino que, además, no existen indicios de que otros Estados vayan a dar el paso de forma inminente. Por eso, no resulta superfluo que una parte importante del Informe esté dedicada a poner de relieve argumentos de distinta naturaleza que exigen una posición más activa, "coercitiva" e inmediata por parte de la UE.

5. En primer lugar, existen razones de derechos humanos y compromisos adquiridos. En efecto, tanto los Estados miembros como la propia UE forman parte de la Convención de las Naciones Unidas sobre los Derechos de las Personas con Discapacidad, de 13 de diciembre de 2006 (en adelante, CDPD). Con ello, asumieron el compromiso de adoptar medidas para proporcionar acceso a las personas con discapacidad al apoyo que puedan necesitar en el ejercicio de su capacidad jurídica ${ }^{3}$. Como bien apunta el Informe, el acceso a los apoyos tiene que quedar garantizado no solo en las situaciones puramente domésticas sino también en las situaciones con elemento extranjero.

6. En segundo lugar, desde el punto de vista sociológico, existe cada vez más movilidad transfronteriza en la esfera de estas personas vulnerables, que pueden propiciar que surjan los problemas propios del derecho internacional privado.

7. En tercer lugar, desde el punto de vista jurídico, existen importantes diferencias entre países en la regulación de la protección de las personas vulnerables, tanto desde el punto de vista sustantivo como procesal.

\footnotetext{
${ }^{1}$ El Informe puede consultarse en https://www.europeanlawinstitute.eu/projects-publications/completed-projects-old/ protection-of-adults/

${ }^{2}$ Alemania, Austria, Chipre, Estonia, Finlandia, Francia, Letonia, Portugal, Reino Unido (solo para Escocia) y República Checa.

${ }^{3}$ Art. 12.3 CDPD. La Observación General N.1 del Comité aclaró que el término capacidad jurídica incluía la capacidad de obrar. El art. 12.4 CDPD añade que los Estados parte se obligaron a asegurar "que en todas las medidas relativas al ejercicio de la capacidad jurídica se proporcionen salvaguardias adecuadas y efectivas para impedir los abusos de conformidad con el derecho internacional en materia de derechos humanos. Esas salvaguardias asegurarán que las medidas relativas al ejercicio de la capacidad jurídica respeten los derechos, la voluntad y las preferencias de la persona, que no haya conflicto de intereses ni influencia indebida, que sean proporcionales y adaptadas a las circunstancias de la persona, que se apliquen en el plazo más corto posible y que estén sujetas a exámenes periódicos por parte de una autoridad o un órgano judicial competente, independiente e imparcial. Las salvaguardias serán proporcionales al grado en que dichas medidas afecten a los derechos e intereses de las personas."
} 
8. En cuarto lugar, esas mismas diferencias se advierten también en la normativa de derecho internacional privado.

9. De la combinación de todos estos elementos, se sigue que la continuidad de la protección de los adultos vulnerables no está garantizada, ya que las medidas que se adopten en un país pueden no ser reconocidas en otro. El cruce de la frontera puede conllevar un cambio de ley aplicable, ley que puede ser incompatible con las medidas adoptadas previamente.

10. Conectando con el derecho de la UE, esto puede provocar distorsiones en la libre circulación de los ciudadanos. La falta de certidumbre en relación con las medidas de protección puede desincentivar el cambio de residencia u otro tipo de movilidad (incluida la de los bienes del adulto). Esto puede constituir al mismo tiempo una discriminación vedada por los Tratados. Incluso pueden verse afectados los derechos de los terceros que contratan con estas personas vulnerables, que no pueden prever si los negocios que celebren son válidos o no.

11. Son varios principios y políticas de la UE los que parecen exigir un paso al frente: el respeto de los derechos fundamentales; el desarrollo del espacio de libertad, seguridad y justicia; el buen funcionamiento del mercado interior; y el principio de subsidiariedad (en la medida en que el objetivo de armonización difícilmente se puede conseguir sin la intervención de la UE).

\section{Bases competenciales para actuar}

12. Otro asunto conflictivo es el de la base competencial de la UE para tomar medidas en este ámbito. La UE carece de competencia para llevar a cabo una armonización desde el punto de vista del derecho sustantivo, ya que el núcleo de la normativa no estaría encaminado al desarrollo del mercado interior (art. 26 TFUE), que sería lo que legitimaría a la UE para ello (sobre la base del art. 114 TFUE). Por ello, el Informe apuesta por una armonización de las normas de derecho internacional privado, con base en el art. 81 TFUE, es decir, en la cooperación judicial en materia civil que incluye la adopción de medidas en situaciones con implicaciones transfronterizas. Estas medidas pueden buscar el reconocimiento mutuo de decisiones judiciales y extrajudiciales, la compatibilidad de normas de ley aplicable y competencia judicial internacional. Por tanto, este precepto permite a la Unión Europea adoptar un Reglamento sobre los aspectos de derecho internacional privado en materia de protección de adultos. A los efectos de la posible adopción de un Reglamento, el Informe aclara que la protección de adultos no se debe considerar una materia de derecho de familia, que precisaría una tramitación especial al amparo del art. 81.3 TFUE (que el Consejo se pronuncie por unanimidad, previa consulta al Parlamento).

13. El fundamento en el art. 81 TFUE es más adecuado que el del 21 TFUE (libre circulación de personas) ya que este último solo justificaría medidas a favor de los ciudadanos de la UE, mientras que las iniciativas presentadas pretenden beneficiar también a quienes no son ciudadanos, ya que se trata de una cuestión de derechos humanos y, por tanto, universal. En todo caso, como bien señala el Informe, los pasos que se den redundarán en beneficio de la libre circulación de personas.

14. Además de la posibilidad de elaborar un Reglamento, el art. 216 TFUE ofrece una base para complementar esta competencia hacia el exterior. El mencionado artículo permite a la UE concluir Convenios internacionales y entablar relaciones internacionales en relación con la protección de los adultos, lo que puede favorecer la armonización de las normas de derecho internacional privado en esta materia. No obstante, la UE no puede ser parte del $\mathrm{CH} / 2000$ ya que el propio Convenio lo impide en sus arts. 53 y 54, que solo permiten la incorporación a Estados soberanos y no a organizaciones internacionales. En estos casos, lo que suele hacer la UE -y es lo que los autores del Informe proponen- es autorizar a los Estados miembros para que ratifiquen el instrumento en cuestión "en interés de la Unión". Como indica el Informe, desde el punto de vista del derecho internacional, el instrumento será vinculante para los Es- 
tados, generando derechos y obligaciones, mientras que, para el derecho de la UE, el Convenio pasaría a formar parte del acervo comunitario.

\section{Estrategia externa}

15. Los redactores del Informe tienen claro que cualquier actuación encaminada a mejorar la protección transfronteriza de los adultos debe tomar como punto de partida el $\mathrm{CH} / 2000$. En primer lugar, porque, pese a ser anterior, el $\mathrm{CH} / 2000$ se asienta en los mismos principios de derechos humanos que el CDPD (entre otros, el respeto a la autonomía y a la dignidad del adulto), principios que también deberá respetar cualquier acción de la UE. En segundo lugar, porque el balance de estos primeros 20 años de funcionamiento del Convenio se pueden considerar positivos. En tercer lugar, porque es conveniente que la armonización alcance al mayor número de países posible.

16. Por ello, las dos propuestas que realiza el Informe giran en torno al Convenio de La Haya de 2000: por un lado, instar a los Estados miembros a su ratificación y promoverla entre terceros Estados; por otro, elaborar un Reglamento que, dentro de su ámbito de aplicación, mejore la eficacia del Convenio entre los Estados miembros de la UE. Por tanto, el Informe recomienda hacer uso tanto de las competencias externas como de las competencias internas de la Unión.

17. En cuanto a las competencias externas, y teniendo en cuenta que el $\mathrm{CH} / 2000$ solo está abierto a los Estados soberanos, la UE debe, por un lado, dar los pasos necesarios para que todos los Estados miembros lo ratifiquen (por el momento solo 9 lo han hecho). Por otro lado, la UE debe promover su ratificación por terceros países, de forma que la protección de los adultos no se limite a situaciones conectadas con Estados de la UE, sino también con terceros países. Esto puede ser especialmente importante a la hora de garantizar el reconocimiento de los poderes otorgados por un adulto en previsión de su futura incapacidad.

18. En relación con el primer punto, y ante la actual pasividad de los Estados miembros (pese a que la UE ya ha hecho diversas llamadas animando a la ratificación), se insta a la UE a adoptar una posición más vinculante. En este sentido, la UE debería autorizar a los Estados miembros que todavía no lo han hecho a ratificar el Convenio en interés de la Unión. Está legitimada para ello en la medida en que está dentro del ámbito de sus competencias externas la ratificación de un Convenio de estas características (aunque, como se ha reiterado, en el caso concreto lo impide el Convenio). En esa medida, los redactores del Informe afirman que, si la UE adoptara una decisión otorgando la autorización para la ratificación en interés de la Unión, esto obligaría a los Estados miembros a llevarla a cabo.

19. Este es uno de los puntos más controvertidos del Informe, dado que sería la primera vez que la UE da este paso en una materia en la que todavía no ha legislado. Pese a ello, consideran que tal acción estaría amparada por el art. 216 TFUE en la medida en que es necesario para avanzar en varios de los objetivos establecidos por los Tratados, pues haría más efectiva la protección de los derechos fundamentales de los adultos, removería obstáculos para la libre circulación de estos, combatiría la exclusión social y la discriminación y promovería la solidaridad intergeneracional. La diversidad de normas de derecho internacional privado pone en riesgo estos objetivos, por lo que la ratificación del Convenio, que conduciría a la uniformidad, favorece su consecución.

20. Otra de las situaciones en las que el art. 216 TFUE permite a la UE ejercitar esta competencia exterior es que se puedan ver afectadas normas de la Unión. Como explica el Informe, aunque no existen actualmente normas de derecho internacional privado en este sector, sí que es cierto que algunas de las normas de derecho internacional privado europeo tocan tangencialmente cuestiones relacionadas con la capacidad (por ejemplo, el art. 13 del RRI). 
21. El Informe ofrece también argumentos frente a otras objeciones que se han hecho a la ratificación de la $\mathrm{CH} / 2000$, como pueden ser las repercusiones financieras -que los autores demuestran que no serían excesivas, dado que el trabajo extra que puede generar para las autoridades centrales es mínimo- y que podría perjudicar el papel actual que los cónsules ejercen para la protección de los adultos en el extranjero (el Informe afirma, por el contrario, que estos seguirán desempeñando un cometido importante).

\section{Estrategia interna}

22. El Informe propone, como estrategia paralela, la adopción de un Reglamento que mejore, entre los Estados miembros, lo establecido por el Convenio. Varias cosas hay que aclarar al respecto.

23. En primer lugar, el establecimiento de unas normas que, siguiendo los principios y objetivos del Convenio, mejoren su aplicación, no supone un quebrantamiento de las obligaciones que los Estados han adquirido o adquirirán con la ratificación del Convenio. En efecto, el propio Convenio prevé la posibilidad de que haya Estados que adopten disposiciones particulares para estrechar la cooperación en sus relaciones mutuas yendo más allá de lo previsto por el Instrumento.

24. El texto propuesto complementaría el $\mathrm{CH} / 2000$ para los Estados miembros de la UE en sus relaciones mutuas. Como afirma de forma clara el Informe, el CH/2000 funcionaría como la regulación general que, en casos intra-europeos, es decir, en casos que afecten a la persona o los bienes de un adulto cuya residencia habitual está en un Estado miembro o caso que de algún otro modo solo estén vinculados con Estados miembros, se aplicaría junto con el Reglamento, que podría complementarlo o introducir matizaciones (art. $49 \mathrm{CH} / 2000)^{4}$.

25. En segundo lugar, en cuanto al posible contenido de estas "mejoras", los redactores reconocen que, pese a percibir que el $\mathrm{CH} / 2000$ tiene algunos puntos que han podido quedar obsoletos, sin embargo, dada la poca experiencia práctica acumulada, no es fácil valorar hasta qué punto las carencias percibidas son tales. Por otro lado, uno de los aspectos que se consideran "mejorables" es la falta de reglas más específicas en algunas cuestiones. Sin embargo, no hay seguridad de que esta falta de detalle pueda aportar flexibilidad y sea, por tanto, positiva.

\section{Contenido de la Propuesta de Reglamento}

26. De lo dicho anteriormente, se deducen las dos líneas maestras de la propuesta de Reglamento que contiene el Informe: por un lado, recoger solo aquello que añada valor al Convenio y, por otro, que no altere su normal funcionamiento. Como se ha dicho, el propio Convenio considera que no afectan a su funcionamiento acuerdos que celebren entre sí Estados contratantes en la medida en que sean aplicables a adultos con residencia habitual en esos Estados. Los autores consideran que tampoco afecta al normal funcionamiento del Convenio la aplicación de normas de reconocimiento de medidas distintas cuando estas procedan de un Estado con el que se ha celebrado un acuerdo. O cuando se decline o asuma competencia, con base en el Reglamento, a favor o en perjuicio de otro Estado miembro que sería competente / incompetente con base en el Convenio.

27. Por otro lado, es importante que todos los Estados miembros apliquen de modo uniforme el Convenio. En todos aquellos aspectos que el Convenio establece la necesidad de que los estados adopten medidas para implementarlo. Este es otro de los aspectos que el Reglamento podría abordar. Se conseguirá así garantizar la uniformidad en la aplicación que la UE busca en todos sus textos de derecho internacional privado.

\footnotetext{
${ }^{4}$ En concreto, el art. 49.2 permite que los Estados contratantes celebren acuerdos respecto de los adultos que residan en alguno de los Estados parte en esos acuerdos
} 
28. El resto del Informe se centra principalmente en exponer y comentar, artículo por artículo, la propuesta de Reglamento. En el preámbulo del nuevo texto se dejaría claro que no se trata de una regulación ex novo de la protección del adulto, sino que se toma como punto de partida el $\mathrm{CH} / 2000$, del que constituye un complemento para su funcionamiento en las relaciones entre los Estados Miembros. El objetivo es que, en estas situaciones intra-europeas, se llegue más lejos en las metas que el propio $\mathrm{CH} / 2000$ persigue.

29. En cuanto a su ámbito material coincide con el del Convenio, y en sus soluciones se alinean con él. Una expresión que el Reglamento añade en la definición del ámbito de aplicación es que este se aplicará en materia "civil-mercantil". Aunque el Informe no comenta nada al respecto, pueden surgir problemas a la hora de calificar algún tipo de medida en las que intervengan autoridades administrativas.

30. En el sector de la competencia judicial internacional, se introduce un precepto para permitir que el adulto que otorgue poderes de representación en previsión de su futura incapacidad pueda decidir que las autoridades del país cuya ley regirá los poderes tengan competencia para su protección. El precepto propuesto por el Informe es coherente con el principio de respeto de los deseos del adulto que tanto el CH/2000 como el CDPD proclaman. En todo caso, esta regla está sujeta a diversas salvaguardias para evitar abusos, que van desde una limitación de las posibilidades de elección (solo las autoridades del país cuya ley sea aplicable a los poderes) a los requisitos estrictos de validez formal. Para que este precepto sea aplicable es necesario que el adulto tenga su residencia habitual en un Estado miembro en el momento de presentación de la demanda. Hay que recordar que el Convenio solo se refería al foro de la autonomía de la voluntad al enumerar las posibles autoridades que podrían recibir la transferencia de la competencia por parte de las autoridades de la residencia habitual (art. $8 \mathrm{CH} / 2000$ ). Con el precepto del Convenio no quedaba garantizado que las autoridades señaladas por el adulto terminasen conociendo del asunto.

La competencia basada en la autonomía de la voluntad no será exclusiva y estas autoridades podrán requerir a otras señaladas en el art. $8 \mathrm{CH} / 2000$ que adopten medidas de protección.

31. En el sector de la eficacia extraterritorial de decisiones, es legítimo y no atenta contra los compromisos adquiridos por los Estados miembros el establecer un régimen más favorable al reconocimiento cuando la decisión proceda de otro Estado miembro. Dado el principio de confianza mutua que rige entre los Estados miembros, la propuesta de reglamento excluye el motivo de denegación previsto en el art. 22.2 a) $\mathrm{CH} / 2000$, a saber, el control de competencia, que permite denegar el reconocimiento cuando la competencia de la autoridad de origen no esté basada en alguno de los foros previstos en el Convenio.

32. Otras mejoras que se proponen para este sector son la previsión de un procedimiento uniforme para obtener la declaración de ejecutabilidad y la creación de un certificado que pueda emitir la autoridad que dicta la medida que facilite la comprobación de determinados extremos y simplifique así el reconocimiento, tal y como se hace en otros instrumentos comunitarios.

33. De igual forma, se considera adecuado establecer la aceptación y la ejecutividad de instrumentos públicos emitidos por un Estado miembro con fines de protección, reconociéndoles el mismo valor probatorio que se le concede en el país de origen.

34. Reviste también interés la propuesta de creación de un Certificado Europeo de Poderes de Representación. Se inspira en el Certificado Sucesorio Europeo y puede facilitar mucho las cosas a quienes representan a los adultos, sirviendo de instrumento probatorio de la existencia y alcance de sus poderes. Este Certificado tendría eficacia en todos los Estados miembros sin necesidad de ningún procedimiento especial.

35. Finalmente, en el ámbito de la cooperación de autoridades, el texto propuesto aboga por la comunicación directa entre autoridades. Son muchas las previsiones que el CH/2000 contiene sobre co- 
municaciones entre autoridades, por lo que la regla propuesta agilizaría mucho los trámites y mejoraría la protección.

\section{Otras propuestas de interés}

36. Aunque no se incluye en la propuesta de Reglamento, el grupo redactor propone la creación de registros nacionales electrónicos de mandatos de protección.

37. También al margen de la propuesta de Reglamento, el Informe contiene una interesante Checklist con los aspectos más importantes que debe recoger un mandato de protección para desplegar todos sus efectos y para evitar problemas de eficacia extraterritorial.

\section{Sugerencias para la discusión con vistas a la Comisión especial sobre el Convenio de Adultos}

38. El Informe también contiene algunas sugerencias para el debate que tendrá lugar con ocasión de la Comisión especial sobre el Convenio de Adultos en 2022. Cabe destacar las interesantes reflexiones sobre el modo de determinar la residencia habitual a los efectos del $\mathrm{CH} / 2000$, teniendo en cuenta que hay casos en los que el adulto carece de libertad para decidir su residencia o no es libre de abandonar el país en el que se encuentra.

39. Otro concepto esencial que requeriría clarificación es el de "medida de protección" y el de "poderes de representación otorgados por el adulto", teniendo en cuenta la heterogeneidad existente que, probablemente, se vaya agravando en la medida en que los países vayan adaptando sus legislaciones al CDPD.

40. También deberán abordarse algunas lagunas existentes. En primer lugar, la determinación de la ley aplicable a los poderes de representación nacidos ex lege. En segundo lugar, se echa en falta una regulación más detallada del ejercicio de la autonomía de la voluntad para elegir la ley aplicable a los mandatos de protección. Finalmente, se pone de relieve la conveniencia de afrontar los problemas de adaptación que pueden surgir con ocasión del reconocimiento de medidas de protección desconocidas acordadas en otro país.

\section{Conclusiones}

41. El Instituto de Derecho Europeo ha realizado una labor encomiable y muy oportuna. Era preciso un trabajo de estas características para mostrar a la Unión Europea la necesidad de tomar alguna decisión en relación con la protección internacional del adulto vulnerable. Los avances que ha propiciado la CDPD tienen que tener también su reflejo en las situaciones transfronterizas, donde el adulto se encuentra en una posición aún más vulnerable.

42. Resulta también acertado tomar como punto de partida el $\mathrm{CH} / 2000$. El escaso número de ratificaciones en comparación con el paralelo CH/1996, sobre protección de los niños, no debe poner en duda la calidad del instrumento. Es más bien la falta de conciencia sobre la importancia de esta realidad y el temor a asumir compromisos lo que, por ahora, paraliza a los Estados para dar el paso de la ratificación. Por eso, es fundamental recordar que no solo es conveniente, sino casi una exigencia para la Unión Europea avanzar en este campo.

43. En cuanto a las soluciones contenidas en la Propuesta de Reglamento, cabe destacar el impulso a la autonomía de la voluntad, que se manifiesta en la promoción de los mandatos de representación y en la introducción, con un papel muy relevante, del foro de la autonomía de la voluntad en un ámbito en el que hasta ahora le parecía vedado. 